\title{
Evaluation of the Balance and Variation of DEMATELs by Using Liu's Integrated Validity Index
}

\author{
Hsiang-Chuan Liu ${ }^{1}$, Wei-Sung Chen ${ }^{2}$, \\ Wei-Guang Tsaur ${ }^{3}$, Hung-Ming Yeh $^{4}$, Shih-Shiunn Chen $^{5}$,Yu Seng Tien ${ }^{6}$, Chin-Long \\ $\mathrm{Hsu}^{7}$ \\ ${ }^{13,4,5,6,7}$ Graduate School of Business Administration, Fu Jen Catholic University. New Taipei, Taiwan, \\ ${ }^{2}$ Department of Computer Science and Information Engineering, Asia University, Taichung, Taiwan \\ ${ }^{3}$ Department of Economics, Fu Jen Catholic University, New Taipei, Taiwan \\ ${ }^{4}$ Department of Accounting, Fu Jen Catholic University, New Taipei, Taiwan
}

\begin{abstract}
In this paper, for evaluating and comparing the heterogeneous balance-variation order pair of any two decision-making trial and evaluation laboratory (DEMATEL) theories, in which one has larger balance and smaller variation, and on the contrary, the other one has smaller balance and larger variation, the first author proposed a useful integrated validity index to evaluate any DEMATEL theory by combining Liu's balanced coefficient and Liu's variation coefficient .Using this new validity index, three kinds of DEMATELs with a same direct relational matrix, including the traditional DEMATEL, shrinkage DEMATEL and balance DEMATEL, are compared, a simple validity experiment is conducted, the results show that the balance DEMATEL has the best performance, the performance of the shrinkage coefficient is better than that of the traditional DEMATEL.
\end{abstract}

Key words:DEMATEL, indirect relational matrix, balance coefficient, variation coefficient, validity index.

\section{Introduction}

Decision Making Trial and Evaluation Laboratory (DEMATEL) was developed between 1972 to 1979 by Science and Human Affairs Program of the Battelle Memorial Institute of Geneva [1]. It can be used to resolve complex and difficult problems in the world, and it has been widely used as one of the best tools to solve the cause and effect relationship among the evaluation factors [1-4]. But the indirect relation of a traditional DEMATEL is always far greater than its direct relation, because the indirect relation matrix does not normalized as that of the direct relational matrix, the traditional DEMADEL is unfair and inaccuracy [5-6], and two improved DEMATELs, the shrinkage DEMATEL and the balanced DEMATEL were proposed, in which the balanced coefficient and variation coefficient are provided, the balanced coefficient can be used to evaluate the balance degree between indirect influences and direct influences of the DEMATEL. If the indirect influences and direct influences of the DEMATEL are balanced, the variation coefficient can be used to evaluate the variation degree of the relation prominence

1 *Corresponding author. E-mail address: Ihc@asia.edu.tw

Tel : +886-2-29096938; Fax: +886-4-2213520

2 james@asia.edu.tw, 3 tsaur66@hotmail.com,4 012939@mail.fju.edu.tw, 5 sschen0604@gmail.com, 6 sparktien@gmail.com, 7 longhsu@gmail.com 
matrix of the DEMATE. We know that the traditional DEMATEL always has smaller balance coefficient and larger variation coefficient, since the indirect influence is larger, and we want to obtain larger balance coefficient and larger variation coefficient. However, how to evaluate the heterogeneous balance-variation order pair of any two DEMATEL theories, in which one has smaller balance and larger variation, and on the contrary, the other one has larger balance and smaller variation, that is a critical issue, in this paper, the first author proposed a useful integrated validity index to evaluate any DEMATEL theory by combining Liu's balanced coefficient and Liu's variation coefficient, because the balance degree is more important than variation degree, when combining the balance coefficient and variation coefficient in the new validity index, we must give the larger weighted score to the balance coefficient than which of variation coefficient, fortunately, the balance coefficient is already more sensitive than variation coefficient, to take their average is well done.

This paper is organised as follows. Section 2 introduces the traditional DEMATEL. Section 3 introduces two improved DEMATELs: the shrinkage DEMATEL and the balanced DEMATEL. Section 4 describes Liu's balanced coefficient, Liu's variation coefficient and the proposed validity index. Section 5 describes the Experiment for comparing three kinds of DEMATELs by using validity index. Finally, Section 6 concludes the paper.

\section{The traditional DEMATEL}

Definition 1. The traditional DEMATEL

The procedure of the traditional DEMATEL method is briefly introduced below: [1-4]

\subsection{Calculate the initial direct relation matrix $Q$}

$\mathrm{N}$ experts are asked to evaluate the degree of direct influence between two factors based on pair-wise comparison. The degree to which the expert e perceived factor $\mathrm{i}$ effects on factor $\mathrm{j}$ is denoted as

$$
q_{i j}^{(e)}, e=1,2, \ldots, N, q_{i j}^{(e)} \in\{0,1,2,3,4\}, i, j=1,2, \ldots, n
$$

For each expert e, an individual direct relation matrix is constructed as

$$
Q_{e}=\left[q_{i j}^{(e)}\right]_{n \times n}, e=1,2, . ., N, q_{i i}^{(e)}=0, i=1,2, \ldots, n
$$

We can obtain their average direct relation matrix, called the initial direct relation matrix $Q$ as follows:

$$
Q=\left[q_{i j}\right]_{n \times n}=\frac{1}{N} \sum_{e=1}^{N} Q_{e}, q_{i j}=\frac{1}{N} \sum_{e=1}^{N} q_{i j}^{(e)}, i, j=1,2, \ldots, . n
$$

\subsection{Calculate the direct relation matrix $\mathrm{A}$}




$$
\begin{gathered}
A=\left[a_{i j}\right]_{n \times n}=\lambda^{-1} Q, \lambda=\max _{1 \leq i, j \leq n}\left\{\sum_{j=1}^{n} q_{i j}, \sum_{i=1}^{n} q_{i j}\right\} \\
a_{i i}=0, i=1,2, \ldots, n, \quad 0 \leq a_{i j} \leq 1, i \neq j, i, j=1,2, \ldots, n, \quad 0 \leq \sum_{i=1}^{n} a_{i j}, \sum_{j=1}^{n} a_{i j} \leq 1, i, j=1,2, \ldots, n
\end{gathered}
$$

\subsection{Calculate the indirect relation matrix $B$ and the total relation matrix $\mathbf{T}$}

Based on Markov chain theory, we have $\lim _{k \rightarrow \infty} A^{k}=0_{n \times n}$

The indirect relation matrix $B=\left[b_{i j}\right]_{n \times n}=\lim _{k \rightarrow \infty}\left[A^{2}+A^{3}+\ldots+A^{k}\right]=A^{2}(I-A)^{-1}$

The total relation matrix $T=\left[t_{i j}\right]_{n \times n}=A+B=\left[\left(a_{i j}+b_{i j}\right)\right]_{n \times n}$

\subsection{Calculate the relation degree and prominence degree of each factor}

$$
r_{i}=\sum_{j=1}^{n} t_{i j}, \quad c_{i}=\sum_{k=1}^{n} t_{k i}, \quad i=1,2, \ldots, n
$$

The value of $r_{i}$ indicates the total dispatch both directly and indirectly effects, that factor $\mathrm{i}$ has on the other factors, and the value of $c_{i}$ indicates the total receive both directly and indirectly effects, that factor $i$ has on the other factors.

The relation degree of factor $\mathrm{i}$ is denoted as $x_{i}=r_{i}-c_{i}, \quad i=1,2, \ldots, n$

The prominence degree of factor $\mathrm{i}$ is denoted as $y_{i}=r_{i}+c_{i}, i=1,2, \ldots, n$

Relation prominence matrix is denoted as $\left(x_{i}, y_{i}\right)_{i=1}^{n}$

\subsection{Set the threshold value ( $\alpha$ )}

For eliminating some minor effects elements in matrix $\mathrm{T}$ to find the impact-relations map, Yang et al. [3] suggest their threshold value below:

$$
\alpha_{Y}=\frac{1}{n^{2}} \sum_{i=1}^{n} \sum_{j=1}^{n} t_{i j}
$$

Lia and Tzeng [4] suggested a more information threshold value, ${ }^{\alpha_{M}}$, based on their maximum mean de-entropy (MMDE) algorithm.

\subsection{Build a cause and effect relationship diagram}


If $t_{i j}>\alpha_{Y}$, or $t_{i j}>\alpha_{M}$, then factor $\mathrm{i}$ is a net dispatch node of factor $\mathrm{j}$, and factor $\mathrm{j}$ is a net receive node of factor $i$, and denoted as

$$
\left(x_{i}, y_{i}\right) \rightarrow\left(x_{j}, y_{j}\right), \text { or }\left(x_{i}, y_{i}\right) \leftarrow\left(x_{j}, y_{j}\right)
$$

The graph of $\left(x_{i}, y_{i}\right)_{i=1}^{n}$ including the net direct edges can present a cause and effect relationship diagram.

\section{The improved DEMATELs}

Two improved DEMATELs: the shrinkage DEMATEL and the balanced DEMATEL are briefly introduced below: [5-6]

\subsection{The shrinkage DEMATEL}

Our previous paper [2] pointed out that the indirect relation of a traditional DEMATEL is always far greater than its direct relation, that is unbalanced and unfair, because the indirect relation matrix does not normalized as the direct relation matrix.

For overcoming this drawback, an external shrinkage coefficient of the indirect relation matrix, d, was provided to construct a better indirect relation matrix, and a generalized DEMATEL theory is obtained below:

\section{Definition 2. The shrinkage DEMATEL [5]}

Let $A=\left[a_{i j}\right]_{n \times n}$ and $B=\left[b_{i j}\right]_{n \times n}$ are defined as Definition 1. The indirect relation matrix with shrinkage coefficient $d$

$$
B_{d}=\left[b_{i j}^{(d)}\right]_{n \times n}=d A^{2}(I-d A)^{-1}, d \in\left[\frac{1}{2}, 1\right]
$$

The total relation matrix with shrinkage coefficient $\mathrm{d}$

$$
T_{d}=\left[t_{i j}^{(d)}\right]_{n \times n}=A+B_{d}=\left[\left(a_{i j}+b_{i j}^{(d)}\right)\right], d \in\left[\frac{1}{2}, 1\right]
$$

The Relation-Prominence of the DEMATEL $\left(A, B_{d}\right)$ with shrinkage coefficient $\mathrm{d}$ is defined as

$$
\begin{aligned}
& R\left(A, B_{d}\right)=\left(x_{i}^{(d)}, y_{i}^{(d)}\right)_{i=1}^{n}=\left(r_{i}^{(d)}-c_{i}^{(d)}, r_{i}^{(d)}+c_{i}^{(d)}\right)_{i=1}^{n} \\
& \text { where } r_{i}^{(d)}=\sum_{j=1}^{n} t_{i j}^{(d)}, c_{i}^{(d)}=\sum_{k=1}^{n} t_{i j}^{(d)}, \quad i=1,2, \ldots, n
\end{aligned}
$$

If $\mathrm{d}=1$, the new DEMATEL $\left(A, B_{d}\right)$ is just the traditional DEMATEL $(A, B)$. If $\mathrm{d}=0.5$, then $\max _{1 \leq i, j \leq n}\left\{\sum_{j=1}^{n} b_{i j}^{(d)}, \sum_{i=1}^{n} b_{i j}^{(d)},\right\} \leq 1$, and the new DEMATEL $\left(A, B_{d}\right)$ is feasible, since its indirect relation influence is no longer greater than its direct relation influence. 


\subsection{The balanced DEMATEL $L$}

Definition 3. The balanced DEMATEL [6]

Let $A=\left[a_{i j}\right]_{n \times n}$ and $B=\left[b_{i j}\right]_{n \times n}$ are defined as Definition 1 .

$$
\mu=\max _{1 \leq i, j \leq n}\left\{\sum_{j=1}^{n} b_{i j}, \sum_{i=1}^{n} b_{i j}\right\}
$$

The normalized indirect relation matrix, $B_{N}$ is defined by

$$
B_{N}=\left[b_{i j}^{(N)}\right]_{n \times n}=\mu^{-1} B=\left[\left(\mu^{-1} b_{i j}\right)\right]_{n \times n}
$$

Normalized total relation matrix is defined as

$$
T_{N}=\left[t_{i j}^{(N)}\right]_{n \times n}=A+B_{N}=\left[\left(a_{i j}+\mu^{-1} b_{i j}\right)\right]_{n \times n}
$$

The Relation-Prominence of the $\operatorname{DEMATEL}\left(A, B_{N}\right)$ is defined as

Where

$$
\begin{aligned}
R\left(A, B_{N}\right) & =\left(x_{i}^{(N)}, y_{i}^{(N)}\right)_{i=1}^{n}=\left(r_{i}^{(N)}-c_{i}^{(N)}, r_{i}^{(N)}+c_{i}^{(N)}\right)_{i=1}^{n} \\
r_{i}^{(N)} & =\sum_{j=1}^{n} t_{i j}^{(N)}, c_{i}^{(N)}=\sum_{i=1}^{n} t_{i j}^{(N)}
\end{aligned}
$$

\section{The validity index of DEMATELS}

Liu's balanced coefficient, Liu's variation coefficient and the proposed validity index are briefly introduced as follows.

\subsection{Liu's balanced coefficient,}

Definition 4. The balanced coefficient [6]

Let $A=\left[a_{i j}\right]_{n \times n}, B=\left[b_{i j}\right]_{n \times n}$ and $\mu=\max _{1 \leq i, j \leq n}\left\{\sum_{j=1}^{n} b_{i j}, \sum_{i=1}^{n} b_{i j}\right\}$ are defined as above, then the balanced coefficient is defined as follows

$$
\beta(A, B)=\frac{2 \sqrt{\mu}}{1+\mu}, \quad 0 \leq \beta(A, B) \leq 1
$$

Note that $\mu=1 \Leftrightarrow \beta(A, B)=1, \mu \neq 1 \Leftrightarrow \beta(A, B)<1$

\subsection{Liu's variation coefficient}

Definition 5. The variation coefficient [5-6] 
Let $A=\left[a_{i j}\right]_{n \times n}, B=\left[b_{i j}\right]_{n \times n}, \quad \mu=\max _{1 \leq i, j \leq n}\left\{\sum_{j=1}^{n} b_{i j}, \sum_{i=1}^{n} b_{i j}\right\} \quad T=\left[t_{i j}\right]_{n \times n}=A+B$, $r_{i}=\sum_{j=1}^{n} t_{i j}, \quad c_{i}=\sum_{k=1}^{n} t_{k i}, \quad i=1,2, \ldots, n$ and $\left(x_{i}, y_{i}\right)_{i=1}^{n}=\left(r_{i}-c_{i}, r_{i}+c_{i}\right)_{i=1}^{n}$ are defined as above.

Then Liu's variation coefficient of the Relation prominence matrix of $(\mathrm{A}, \mathrm{B})$ is defined as:

where

$$
\begin{aligned}
\sigma_{L}(A, B) & =1-\frac{1}{1+5 \sqrt{\sum_{i=1}^{n} \sqrt{\left(x_{i}-\bar{x}_{d}\right)^{2}+\left(y_{i}-\bar{y}_{d}\right)^{2}}}} \\
\bar{x}_{d} & =\frac{1}{n} \sum_{j=1}^{n} x_{i}, \bar{y}_{d}=\frac{1}{n} \sum_{j=1}^{n} y_{i}
\end{aligned}
$$

\subsection{Liu's validity coefficient,}

Definition 6. The variation coefficient [5-6]

If $A=\left[a_{i j}\right]_{n \times n}$ is the direct relation matrix, $B=\left[b_{i j}\right]_{n \times n}$ is the indirect relation matrix, $\beta(A, B)$ is Liu's balance coefficient and $\sigma_{L}(A, B)$ is the variation coefficient, then the variation coefficient is defined as

$$
V_{L}(A, B)=\frac{1}{2}\left[\beta_{L}(A, B)+\sigma_{L}(A, B)\right]
$$

\section{Experiment}

An experiment for Comparing three DEMATELs, by using the validity index is conducted as flows.

Suppose 10 experts are asked to evaluate the degree of direct influence between two factors based on pair-wise comparison. The degree to which the expert e perceived factor i effects on factor $\mathrm{j}$ is denoted as

$$
q_{i j}^{(e)}, e=1,2, . ., 10 \quad q_{i j}^{(e)} \in\{0,1,2,3,4\} \quad i, j=1,2,3,4
$$

For each expert e, an individual direct relation matrix is constructed as

$$
Q_{e}=\left[q_{i j}^{(e)}\right]_{n \times n}, e=1,2, . ., 10, q_{i i}^{(e)}=0, i=1,2,3,4
$$

then we can obtain the initial direct relation matrix Q as follows:

$$
Q=\left[q_{i j}\right]_{4 \times 4}=\frac{1}{10} \sum_{e=1}^{10} Q_{e}, q_{i j}=\frac{1}{10} \sum_{e=1}^{10} q_{i j}^{(e)}, i, j=1,2,3,4
$$

The direct relation matrix A:

$$
A=\left[a_{i j}\right]_{4 \times 4}=\lambda^{-1} Q, \lambda=\max _{1 \leq i, j \leq 4}\left\{\sum_{j=1}^{4} q_{i j}, \sum_{i=1}^{4} q_{i j}\right\}
$$




$$
A=\left[a_{i j}\right]_{4 \times 4}=\left[\begin{array}{cccc}
0 & 0.36 & 0.32 & 0.32 \\
0.32 & 0 & 0.34 & 0.30 \\
0.34 & 0.30 & 0 & 0.30 \\
0.28 & 0.28 & 0.30 & 0
\end{array}\right]
$$

\subsection{DATA for traditional DEMATEL}

The indirect relation matrix, total relation matrix, relation-prominence matrix, balance coefficient, variation coefficient and validity index are listed as follows.

The indirect relation matrix $\mathrm{B}$ :

$$
B=\left[b_{i j}\right]_{4 \times 4}=A^{2}(I-A)^{-1}=\left[\begin{array}{llll}
3.9448 & 3.8483 & 3.9290 & 3.7995 \\
3.7483 & 3.8237 & 3.7991 & 3.6907 \\
3.6807 & 3.6963 & 3.8253 & 3.6331 \\
3.4499 & 3.4478 & 3.4963 & 3.4508
\end{array}\right]
$$

The maximum row and column value of indirect relation matrix:

$$
\mu=\max _{1 \leq i, j \leq n}\left\{\sum_{j=1}^{n} b_{i j}, \sum_{i=1}^{n} b_{i j}\right\}=15.5215 \square \max _{1 \leq i, j \leq n}\left\{\sum_{j=1}^{n} a_{i j}, \sum_{i=1}^{n} a_{i j},\right\}=1
$$

The balance coefficient is $\beta_{L}(A, B)=\frac{2 \sqrt{\mu}}{1+\mu}=0.4769$

The total relation matrix $\mathrm{T}$ :

$$
T=\left[t_{i j}\right]_{4 \times 4}=\left[\begin{array}{llll}
3.9448 & 4.2083 & 4.2490 & 4.1195 \\
4.0683 & 3.8237 & 4.1391 & 3.9907 \\
4.0207 & 3.9963 & 3.8253 & 3.9331 \\
3.7299 & 3.7278 & 3.7963 & 3.4508
\end{array}\right]
$$

The Relation-Prominence matrix of $(A, B)$ :

$$
R(A, B)=\left(x_{i}, y_{i}\right)_{i=1}^{n}=\left[\begin{array}{rr}
0.7579 & 32.2851 \\
0.2658 & 31.7781 \\
-0.2344 & 31.7850 \\
-0.7893 & 30.1989
\end{array}\right]
$$

The variation coefficient of $R(A, B)$ is: $\sigma_{L}(A, B)=0.9015$

\subsection{DATA shrinkage DEMATEL with shrinkage coefficient 0.5}

Indirect relation matrix, total relation matrix, relation-prominence matrix with shrinkage coefficient 0.5 , balance coefficient, variation coefficient and validity index are listed as follows: 


$$
B_{0.5}=\left[\begin{array}{llll}
0.2538 & 0.1993 & 0.2144 & 0.2047 \\
0.2012 & 0.2450 & 0.2002 & 0.2012 \\
0.1916 & 0.2020 & 0.2450 & 0.1971 \\
0.1882 & 0.1879 & 0.1872 & 0.2182
\end{array}\right]
$$

The maximum row and column value of indirect relation matrix with shrinkage 0.5 :

$$
\mu_{0.5}=\max _{1 \leq i, j \leq n}\left\{\sum_{j=1}^{n} b_{i j}^{(0.5)}, \sum_{i=1}^{n} b_{i j}^{(0.5)}\right\}=0.8721<1
$$

The balance coefficient is: $\beta\left(A, B_{0.5}\right)=\frac{2 \sqrt{\mu_{0.5}}}{1+\mu_{0.5}}=0.9977$

The total relation matrix with shrinkage coefficient 0.5 :

$$
T_{0,5}=\left[\begin{array}{llll}
0.2538 & 0.5593 & 0.5344 & 0.5247 \\
0.5212 & 0.2450 & 0.5402 & 0.5012 \\
0.5316 & 0.5020 & 0.2450 & 0.4971 \\
0.4682 & 0.4679 & 0.4872 & 0.2182
\end{array}\right]
$$

The Relation-Prominence matrix of $\left(A, B_{0.5}\right)$ :

$$
R\left(A, B_{0.5}\right)=\left(x_{i}^{(0.5)}, y_{i}^{(0.5)}\right)_{i=1}^{n}=\left[\begin{array}{rr}
0.0973 & 3.6469 \\
0.0334 & 3.5818 \\
-0.0311 & 3.5824 \\
-0.0996 & 3.3826
\end{array}\right]
$$

The variation coefficient of $R\left(A, B_{0.5}\right): \sigma_{L}\left(A, B_{0.5}\right)=0.7653$

The validity index of $\left(A, B_{0.5}\right)$ :

$$
V_{L}\left(A, B_{0.5}\right)=0.8815>V_{L}(A, B)=0.6892
$$

\subsection{DATA of the balanced DEMATEL}

The indirect normalized relation matrix, total normalized relation matrix, normalized relation-prominence matrix, balance coefficient, variation coefficient and validity index are listed as follows:

The normalized indirect relation matrix:

$$
B_{N}=\mu^{-1} B=\left[\begin{array}{cccc}
0.2541 & 0.2479 & 0.2531 & 0.2448 \\
0.2415 & 0.2464 & 0.2448 & 0.2378 \\
0.2371 & 0.2381 & 0.2464 & 0.2341 \\
0.2223 & 0.2221 & 0.2253 & 0.2223
\end{array}\right]
$$

The maximum row and column value of normalized indirect relation matrix: 


$$
\mu_{N}=\max _{1 \leq i, j \leq n}\left\{\sum_{j=1}^{n} b_{i j}^{(N)}, \sum_{i=1}^{n} b_{i j}^{(N)}\right\}=1
$$

The balance coefficient is: $\beta\left(A, B_{N}\right)=\frac{2 \sqrt{\mu_{N}}}{1+\mu_{N}}=1$

The normalized Relation-Prominence matrix of $\left(A, B_{N}\right)$ :

$$
R P_{N}=\left(x_{i}^{(N)}, y_{i}^{(N)}\right)_{i=1}^{n}=\left(\begin{array}{rr}
0.1050 & 3.8950 \\
0.0358 & 3.8249 \\
-0.0338 & 3.8254 \\
-0.1070 & 3.6109
\end{array}\right)
$$

The variation coefficient of $R\left(A, B_{N}\right)$ :

$$
\sigma_{L}\left(A, B_{N}\right)=0.7717>\sigma_{L}\left(A, B_{0.5}\right)=0.7653
$$

The validity index of $\left(A, B_{N}\right)$ :

$$
V_{L}\left(A, B_{N}\right)=0.8859>V_{L}\left(A, B_{0.5}\right)=0.8815>V_{L}(A, B)=0.6892
$$

The results of all of the validity indexes show that the balanced DEMATEL has the best performance, and the performance of the shrinkage DEMATEL with the shrinkage coefficient 0.5 is better than which of the traditional DEMATEL.

\section{Conclusion}

In this paper, the first author points out that if the value of the correlation coefficient of any two items is negative, then the ordering coefficient of IRST for these two items is negative, and the direction of their ordering relation of is revised, this is the reason for which ordering coefficient does not satisfy the Liu's item consistency property, and then, for overcoming this drawback, when the correlation coefficient is negative, we can correct the revised direction by taking the absolved value of the negative ordering coefficient to propose our improved Item Relational Structure Theory, LIRST, this new theory is not only more sensitive than LOT, but also more satisfied Liu's ordering consistence property than LOT.

\section{References}

[1] S. Detcharat \& A. Pongpun, "Using DEMATEL Method to Analyze the Causal Relations on Technological Innovation Capability Evaluation Factors in Thai Technology-Based Firms," International Transaction Journal of Engineering, 81-103, 2013.

[2] G.H. Tzeng, C.H. Chiang, \& C.W. Li, "Evaluating intertwined effects in e-learning programs:A Novel Hybrid MCDM Model Based on Factor Analysis and DEMATEL," Expert Systems with Applications, vol. 32: 1028-1044, 2007. 
[3] Y.P. Yang, H.M. Shieh, J.D. Leu \& G.H. Tzeng, "A novel hybrid MCDM model combined with DEMATEL and ANP with applications," International Journal Operational Research, vol. 5, no. 3:160-168, 2008.

[4] C.W. Lia, G.H. Tzeng, "Identification of a threshold value for the DEMATEL method using the maximum mean de-entropy algorithm to find critical services provided by a semiconductor intellectual property mall," Expert Systems with Applications, vol. 36:9891-9898, 2009.

[5] H.-C. Liu, B.-C. Shia, Y.-C. Ou, H.-W. Su, "A generalized DEMATEL theory with a shrinkage coefficient of indirect relation matrix," IMETI 2015, Kaohsiung Taiwan, 2015.

[6] H.-C. Liu, Y.-C. Ou, H.-C. Tsai, B.-C. Shia, J.-M. Ju, "A balanced DEMATEL theory with normalized indirect relation matrix, " ICICIC 2015, Dalian, China, 2015.

Hsiang-Chuan Liu

Department of Bioinformatics and Medical Engineering

Asia University, Taiwan

lhc@asia.edu.tw 